\title{
CXC chemokine ligand 16, inversely correlated with CD99 expression in Hodgkin Reed-Sternberg cells, is widely expressed in diverse types of lymphomas
}

\author{
FANG LIU $^{1 *}$, YU ZHANG $^{2 *}$, HONGPING TANG $^{2}$, XINHUA ZHOU $^{2}$, \\ ZIQING WU ${ }^{2}$, DONGSHENG TANG ${ }^{1}$ and TONG ZHAO ${ }^{2}$ \\ ${ }^{1}$ Department of Basic Medical Sciences, Medical School, Foshan University, Foshan, Guangdong 528000; \\ ${ }^{2}$ Department of Pathology, Nanfang Hospital, Southern Medical University, \\ Guangzhou, Guangdong 510515, P.R. China
}

Received April 17, 2013; Accepted May 20, 2013

DOI: 10.3892/or.2013.2522

\begin{abstract}
The present study examined a correlation between CXC chemokine ligand 16 (CXCL16) and cell differentiation antigen 99 (CD99) expression and investigated the role of CXCL16 in human lymphoma cell lines and clinical samples. Cytokine antibody arrays were used to measure the differentially expressed cytokines in tumor tissues. The expression of CXCL16 and CD99 was analyzed by quantitative PCR (qPCR) and western blotting, while the pathways involved were assessed by western blotting and enzyme-linked immunosorbent assay (ELISA). The expression of CXCL16 was investigated in 9 lymphoma cell lines (L428, RPMI-8226,
\end{abstract}

Correspondence to: Professor Dongsheng Tang, Department of Basic Medical Sciences, Medical School, Foshan University, 5 Hebing Road, Chancheng, Foshan, Guangdong 528000, P.R. China E-mail: tangdsh@163.com

Professor Tong Zhao, Department of Pathology, Nanfang Hospital, Southern Medical University, 1838 North Guangzhou Dadao, Guangzhou, Guangdong 510515, P.R. China

E-mail: zhaotongketizu@126.com

${ }^{*}$ Contributed equally

Abbreviations: ALCL, anaplastic large cell lymphoma; CD99, cell differentiation antigen 99; cHL, classical Hodgkin lymphoma; CXCL16, CXC chemokine ligand 16; DLBCL, diffuse large B-cell lymphoma; HL, Hodgkin lymphoma; H/RS, Hodgkin ReedSternberg; mCD99L2, mouse CD99 antigen-like 2; MM, multiple myeloma; NHL, non-Hodgkin lymphoma; IL-6, interleukin-6; IL-10, interleukin-10; VCAM-1, vascular cell adhesion molecule 1; MIP-1 $\gamma$, macrophage inflammatory protein- $1 \gamma$; PF4, platelet factor 4; MIG, monokine induced by interferon- $\gamma$; TNF- $\alpha$, tumor necrosis factor- $\alpha$; U-PTL, peripheral T-cell lymphoma, unspecified; RANTES, regulated upon activation normal T-cell expressed and secreted

Key words: chemokine, CXC chemokine ligand 16, classical Hodgkin lymphoma, non-Hodgkin lymphoma, Hodgkin Reed-Sternberg cells
KM3, Jurkat, OCI-Ly1, OCI-Ly8, OCI-Ly10, Karpass299 and Raji) as well as in clinical lymphoma samples using qPCR, western blotting and immunochemistry. Soluble CXCL16 (sCXCL16) levels were measured by ELISA and proliferation was analyzed by CCK-8 proliferation assays. CXCL16 was one of the upregulated chemokines when lymphoma cells where transferred from in vitro to in vivo conditions. The increased expression and secretion of CXCL16 paralleled with a decrease of mCD99L2 and was accompanied by NF- $\kappa$ B pathway activation and vice versa. CXCL16 was expressed in all 9 lymphoma cell lines with the highest levels in the Hodgkin lymphoma (HL) cell line L428, the plasma cell-derived cell lines RPMI-8226 and KM3 and the T leukemia-derived cell line Jurkat. Higher levels of sCXCL16 were secreted by L428 cells, the diffuse large B-cell lymphoma (DLBCL)-derived cell lines (OCI-Ly1, OCI-Ly8 and OCI-Ly10) and Jurkat cells. CXCL16 was widely expressed in clinical samples of lymphoma patients with higher levels in HL compared to non-Hodgkin lymphoma. Human recombinant CXCL16 had no significant effect on L428 cell proliferation, but was able to stimulate $\mathrm{CD}^{+} \mathrm{T}$ lymphocytes to proliferate. CXCL16, inversely correlated with CD99 expression in Hodgkin Reed-Sternberg (H/RS) cells, is widely expressed in diverse types of lymphomas.

\section{Introduction}

In recent years, the association between chronic inflammation and tumor has gained increasing attention (1). Diverse roles have been reported for chemokines in tumor biology, including transformation, proliferation and angiogenesis as well as recruiting lymphocytes to the tumor sites (2). Lymphoma, which can be divided into Hodgkin lymphoma (HL) and non-Hodgkin lymphoma (NHL), is a type of tumor derived from malignant lymphocytes and HL, in particular, is closely related with immune functions and inflammation (3).

Different from NHL, the classical Hodgkin lymphoma (cHL), which is the main HL type, is an unusual type of malignancy in which the clonal B-cell population, known as the Hodgkin Reed-Sternberg (H/RS) cells, contributes to less than $1 \%$ of the tumor tissue. $\mathrm{H} / \mathrm{RS}$ cells survive in 
a large amount of background inflammatory cells, which are composed of lymphocytes, plasma cells, eosinophils, histiocytes and others $(4,5)$. The transformation of H/RS cells has been reported to be related to the loss of cell differentiation antigen 99 (CD99) from B-lymphocytes $(6,7)$ and was recently confirmed by our team (8), who also established the CD99 overexpressing L428 cell line (L428-CD99+) and confirmed that L428-CD99+ cells lost their nature of H/RS cells. Furthermore, mouse CD99 antigen-like 2 (mCD99L2), a widely expressed antigen with moderate sequence homology to human CD99 (9), was investigated by our group in our recently published study (10), in which the stable mCD99L2 downregulated A20 cell line (A20-mCD99L2) was established by lentivirus conducted stable mCD99L2 RNA interference. During the investigation of small hairpin RNA (shRNA) targeting mCD99L2 effects in vitro and in vivo, CXC chemokine ligand 16 (CXCL16) was noted among the differentially expressed cytokines. In the present study, A20-mCD99L2 tumor cells were inoculated into homologous BALB/c mice and the differentially expressed cytokines/ chemokines in tumor tissues were extensively analyzed, and we focused on CXCL16 again.

CXCL16 is a newly found chemokine belonging to the CXC chemokine family $(11,12)$. The human CXCL16 gene is located on chromosome $17 \mathrm{p} 13$ and is normally expressed on the antigen conferring cells (11), occurring both as transmembrane form (TM-CXCL16) as well as soluble form (sCXCL16). The TM-CXCL16 is converted by a disintegrin and metalloproteinase (ADAM) 10 or ADAM 17 into SCXCL16, which is secreted into the supernatants of cell cultures and functions as chemotactic factors for its effective cells (13). High expression of CXCL16 has been associated with a number of human inflammatory diseases, including rheumatoid arthritis, atherosclerosis, interstitial lung diseases, liver injury, coronary artery disease and inflammatory bowel disease (14-17).

CXCL16 was recently reported to be overexpressed in several types of cancer such as colorectal cancer (18), prostate cancer (19) and correlated with lymph node metastasis in epithelial ovarian carcinoma (20) and it may also be involved in the self renewal of tumor stem cells (21). DarashYahana et al (22) mentioned positive CXCL16 expression in some lymphomas, but the types and subtypes have not been clarified. Lymphomas can be classified into more than 80 types or subtypes and the characteristics of CXCL16 in lymphomas require further elucidation. Although CXCL16 was previously noted in our work, in the present study we extended our findings to an association between CXCL16 and CD99 expression, and, in addition, relevant signaling pathways were assessed. Furthermore, the characteristics of CXCL16 expressions in a broad range of human lymphoma cell lines and clinical samples were investigated, which may contribute to further research of the complex lymphoma microenvironments.

\section{Materials and methods}

Ethics statement. All the experimental protocols and human samples obtained from patients undergoing lymphoma treatments were approved by the Clinical Research Ethics Committee of Nanfang Hospital, Southern Medical University.
Case selection. A representative series of $45 \mathrm{cHL}$ and $35 \mathrm{NHL}$ samples, which were diagnosed between 2009 and 2012 and classified according to the World Health Organization (WHO) criteria, were selected from Nanfang Hospital, Southern Medical University, Guangzhou, China (Table I). In addition, 7 reactive and 7 normal lymph node sections were selected as controls. All clinical samples were paraffin-embedded blocks.

Cells and culture. Nine cell lines with different origins and significantly different clinical characteristics were conserved by the Guangdong Provincial Key Laboratory of Molecular Oncopathology and selected for the present study (Table II). The sub-clones of A20 cells transfected with shRNAs targeting mCD99L2 (A20-mCD99L2-) or negative control vectors (A20-CTR) and the sub-clones of L428 cells transfected with the human CD99 gene (L428-CD99+) or negative control vectors (L428-CTR) were previously constructed by our team. Cells were cultured in RPMI-1640 medium supplemented with $10 \%$ heat-inactivated fetal bovine serum (FBS) (HyClone, Logan, UT, USA) at $37^{\circ} \mathrm{C}$ and $5 \% \mathrm{CO}_{2}$.

Immunochemistry analyses. Immunohistochemistry (IHC) of paraffin sections from clinical samples and immunocytochemistry (ICC) of fixed cells from cultured cell lines were carried out, using the ChemMate ${ }^{\mathrm{TM}}$ EnVision $^{\mathrm{TM}}$ Detection kit (Dako, Carpinteria, CA, USA). Heat-induced antigen retrieval was performed in $10 \mathrm{mM}$ citrate buffer ( $\mathrm{pH}$ 6.0) for $2 \mathrm{~min}$ at $100^{\circ} \mathrm{C}$. Endogenous peroxidase activity and non-specific antigen were blocked with peroxidase blocking reagent containing 3\% hydrogen peroxide and serum, followed by incubation with the rabbit anti-human CXCL16 antibody (ab101404, 1:100, Abcam, Cambridge, UK) overnight at $4^{\circ} \mathrm{C}$. After washing, the sections were incubated with biotin-labeled goat anti-rabbit antibody (Zhongshan Inc., Zhongshan, China) for $10 \mathrm{~min}$ at room temperature, and were subsequently incubated with streptavidin-conjugated horseradish peroxidase (HRP) (Maixin Inc., Shenzhen, China). The peroxidase reaction was developed using 3,3-diaminobenzidine chromogen solution in DAB buffer substrate. Sections were visualized with DAB and counterstained with hematoxylin, mounted in neutral gum and analyzed using a bright field microscope.

Evaluations of the immunohistochemical staining results were conducted independently by two independent pathologists (T.Z. and HP.T.) with blinding of clinical data. Staining results were evaluated semi-quantitatively as follow: (-), negative or positive $<10 \%$; (+), moderate positive $10 \%-50 \%$; (++), strong positive $>50 \%$ for intensity of immunostaining.

Immunofluorescence and confocal microscopy were performed as previously described (8). The cells were labeled with goat anti-human CXCL16 (SC-27344, 1:100; Santa Cruz Biotechnology, Santa Cruz, CA, USA) followed by PE-conjugated donkey anti-goat IgG (1:100; Proteintech Group Inc., Chicago, IL, USA). Negative controls were performed by replacing the primary antibodies with PBS.

$R N A$ isolation and quantitative RT-PCR. The extraction of total RNA, generation of cDNA and real-time reverse transcription PCR (RT-PCR) were performed as previously described (10). The following primers were used: CXCL16, 
Table I. The selected samples of different lymphoma types.

\begin{tabular}{lrlr}
\hline Subtype of cHL & $\mathrm{n}$ & \multicolumn{1}{c}{ Subtype of NHL } & n \\
\hline Nodular sclerosis (NSHL) & 12 & Diffuse large B-cell lymphoma (DLBCL) & B \\
Mixed cellularity (MCHL) & 13 & Burkitt's lymphoma (BL) & 5 \\
Lymphocyte-rich (LRCHL) & 15 & Anaplastic large cell lymphoma (ALCL) & Peripheral T-cell lymphoma, unspecified (U-PTL) \\
Lymphocyte-depleted (LRCHL) & 5 & P \\
\hline
\end{tabular}

cHL, classical Hodgkin lymphoma; NHL, non-Hodgkin lymphoma.

Table II. Names and origins of the nine lymphoma cell lines.

\begin{tabular}{ll}
\hline Cell line & \\
\hline L428 & An H/RS cell line from Hodgkin lymphoma (HL) \\
OCI-Ly1, OCI-Ly8, OCI-Ly10 & Three cell lines from diffuse large B-cell lymphoma (DLBCL) \\
Raji & An EB virus positive cell line from Burkitt's lymphoma (BL) \\
Karpass299 & A CD30 positive cell line from anaplastic large cell lymphoma (ALCL) \\
Jurkat & T-leukemia derived cell line \\
RPMI-8226 and KM3 & Two multiple plasma cell lines from multiple myeloma (MM)
\end{tabular}

H/RS, Hodgkin Reed-Sternberg; EB virus, Epstein-Barr virus.

5'-CGTCACTGGAAGTTGTTATTGTGGT-3' (forward) and 5'-TGGTAGGAAGTAAATGCTTCTGGTG-3' (reverse); GAPDH, 5'-ACAGTCAGCCGCATCTTCTT-3' (forward) and 5'-GACAAGCTTCCCGTTCTCAG-3' (reverse). Results were analyzed using the software installed in the 7500 Real-Time PCR system (Applied Biosystems) and the relative expression ratio was determined by the formula $2^{-\Delta \Delta \mathrm{Ct}}$.

Western blot analysis. The western blot analyses were performed as previously described (10). Rabbit anti-human CXCL16 antibody (ab101404, 1:1,000; Abcam), goat anti-mouse CXCL16 antibody (ab84435, 1:500; Abcam) and mouse antihuman $\beta$-actin antibody (TA-09, 1:2,000; Zhongshan) were applied, respectively. Specific binding was correspondingly revealed by HRP-conjugated goat anti-rabbit IgG (ZB-2301, 1:5,000, Zhongshan), rabbit anti-goat IgG (ZB-2306, 1:5,000; Zhongshan) and goat anti-mouse IgG (ZB-2305, 1:5,000), respectively, with an enhanced chemiluminescence system (ECL-Plus, Amersham).

Enzyme-linked immunosorbent assay (ELISA). Secreted CXCL16 concentrations of cell supernatants were measured using commercially available kits (900-M230 Human CXCL16 Mini EDK kit; Mini EDK buffer kit; Peprotech) following the procedures recommended by the manufacturer. The range of sensitivity for human sCXCL16 was $0-2,000 \mathrm{pg} / \mathrm{ml}$.

Isolation of human peripheral blood $C D 4^{+} T$ cells. Peripheral blood mononuclear cells (PBMCs) from healthy donors were prepared using a Lymphocyte Separation kit (Weijia Co., China). $\mathrm{CD}^{+} \mathrm{T}$ cells were isolated using immunomagnetic beads flow cytometry (23). The CD4 ${ }^{+} \mathrm{T}$ cells $\left(2 \times 10^{4}\right.$ cells/well $)$ were cultured with LPS ( $2 \mu \mathrm{g} / \mathrm{ml}$, BD Biosciences) stimulation in anti-CD3-coated plates.

Cell proliferation assays. Cell Counting Kit-8 (CCK-8, Tongren Shanghai Co., Shanghai, China) was used to detect the cell proliferation. $\mathrm{L} 428$ cells or $\mathrm{CD} 4^{+} \mathrm{T}$ cells were seeded in 96-well plates at a density of 1,000 cells/well with $100 \mu \mathrm{l}$ of complete culture medium. After $24 \mathrm{~h}$, the medium was changed to DMEM supplemented with 5\% FBS. Recombinant human CXCL16 (300-55, Peprotech, USA) was added to the medium in final concentrations ranging from 10 to $100 \mathrm{ng} / \mathrm{ml}$. The cells were then cultured for another 1-6 days. Cells not exposed to CXCL16 were used as controls, and wells to which only culture medium was added served as blanks. At the end of the CXCL16 stimulations, the supernatant was removed and $100 \mu \mathrm{l}$ of DMEM medium containing $10 \mu \mathrm{l}$ of CCK- 8 was added to each well for another $3 \mathrm{~h}$ at $37^{\circ} \mathrm{C}$. The culture plates were then shaken for $10 \mathrm{~min}$ and the optical density (OD) values were read at $450 \mathrm{~nm}$.

Animals and in vivo tests. BALB/c mice $(\mathrm{n}=14)(6-8$-week-old female/male) were purchased from the Central Laboratory of Animal Science at the Southern Medical University (Guangzhou) and divided into two groups randomly. Tumor cells $\left(2 \times 10^{7}\right)$ (A20-mCD99L2 ${ }^{-}$or A20-CTR) in $0.1 \mathrm{ml}$ growth medium were injected into the left axillary fossa of the mice subcutaneously. When exhibiting external signs of suffering, the mice were sacrificed and the tumor tissues were conserved in liquid nitrogen. All procedures were conducted under sterile conditions. 
Table III. Cytokines upregulated in excess of 1.5 -fold in tissue from A20-mCD99L2- cell-induced BALB/c mouse tumor compared with cultured A20-mCD99L2 cells.

\begin{tabular}{lrrllllll}
\hline Row & Col & Col & Name & 2-primary & 4-primary & 2-standard & 4-standard & 4-standard/2-standard \\
\hline 1,2 & 13 & $\mathrm{~m}$ & CXCL16 & 14359.5 & 17044.5 & 0.212270472 & 0.387308078 & 1.825 \\
5,6 & 5 & $\mathrm{e}$ & IL-10 & 11541.5 & 15544 & 0.15395003 & 0.345131159 & 2.242 \\
7,8 & 5 & $\mathrm{e}$ & MIG & 9185 & 13276 & 0.105180647 & 0.281380907 & 2.675 \\
7,8 & 7 & $\mathrm{~g}$ & MIP-1 $\gamma$ & 9780 & 32543.5 & 0.11749458 & 0.822962908 & 7.004 \\
7,8 & 10 & $\mathrm{j}$ & MIP-3 $\alpha$ & 9521 & 9629.5 & 0.112134398 & 0.178882982 & 1.595 \\
7,8 & 11 & $\mathrm{k}$ & PF4 & 20683 & 25677 & 0.343139639 & 0.629955366 & 1.836 \\
7,8 & 12 & $\mathrm{l}$ & P-selectin & 29052 & 32292.5 & 0.516341831 & 0.815907655 & 1.5806 \\
7,8 & 13 & $\mathrm{~m}$ & RANTES & 14570.5 & 18204.5 & 0.216637262 & 0.419914027 & 1.9386 \\
9,10 & 10 & $\mathrm{j}$ & VCAM-1 & 11018.5 & 39538.5 & 0.143126187 & 1.019582402 & 7.124 \\
9,10 & 11 & $\mathrm{k}$ & VEGF & 12655 & 13621.5 & 0.176994676 & 0.29109242 & 1.645 \\
\hline
\end{tabular}

2,A20-mCD99L2- cells; 4, A20-mCD99L2- tissue group.
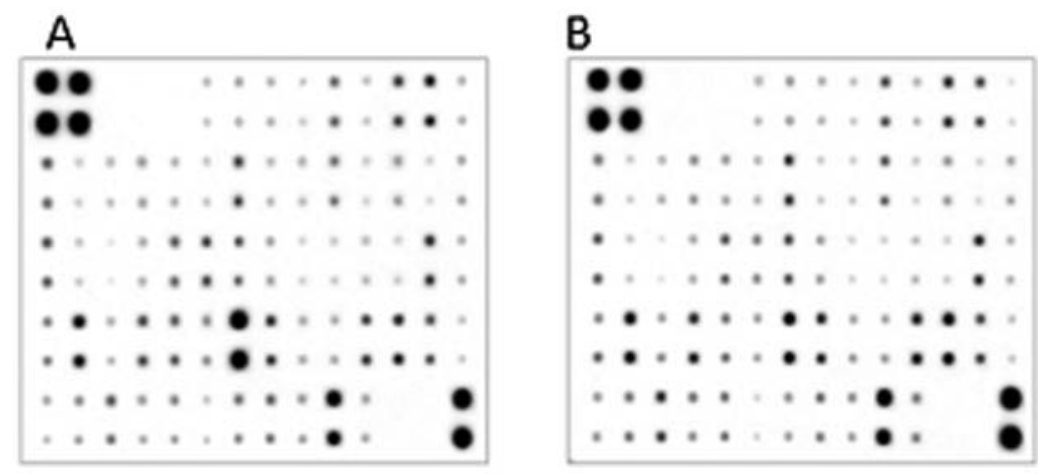

Figure 1. Primary image of mouse antibody array illustrates the cytokine expressions of tumor tissues. (A) A20-CTR group; (B) A20-mCD99L2- group.

Mouse cytokine antibody arrays. RayBiotech mouse cytokine antibody arrays were used for detecting the expression of cytokine/chemokine in tumor tissues from BALB/c mice according to the manufacturer's protocol and as previously described (10). The cytokine expressions were monitored and the differences were analyzed.

Statistical analysis. SPSS 13.0 software was used for statistical analysis. Results are expressed as means $\pm \mathrm{SD}$. One-way ANOVA was used to determine the differences between groups for all in vitro analyses. A P-value of $<0.05$ was considered to indicate a statistically significant result.

\section{Results}

Multiple cytokines are involved in tumor growth in vivo. The tumor tissues exhibited different cytokine/chemokine profiles compared to the corresponding in vitro tumor cell culture conditions (Fig. 1). Compared with the cultured A20-mCD99L2 cells, A20-mCD99L2-induced tumor tissues exhibited upregulated cytokine/chemokine levels, including vascular cell adhesion molecule 1 (VCAM-1), macrophage inflammatory protein- $1 \gamma$ (MIP- $1 \gamma)$, monokine induced by interferon- $\gamma$ (MIG), interleukin-10 (IL-10), regulated upon activation normal T-cell expressed and secreted (RANTES), platelet factor 4 (PF4), and CXCL16 (Table III), whereas the downregulated cytokine/chemokines included interleukin-6 (IL-6), eotaxin and tumor necrosis factor- $\alpha$ (TNF- $\alpha$ ). Among the above mentioned upregulated cytokines/chemokines, CXCL16 caught our attraction, as it was already noted to be upregulated in A20-mCD99L2 cells in our previous study (10) and was now further upregulated in tumor tissues of BALB/c mice inoculated with A20-mCD99L2 cells.

CXCL16 inversely correlates with human/mouse CD99 expression. The mRNA and protein expression of CXCL16 was measured by qRT-PCR and western blot analysis. As CXCL16 can exist as transmembrane form (TM-CXCL16) and soluble form (sCXCL16), supernatants of serum-free cultured cells were collected and the secreted sCXCL16 was quantified by ELISA. In the murine cell lines, results indicated that the expression of mouse CXCL16, as well as SCXCL16, was markedly higher in A20-mCD99L2- cells than in the control (Fig. 2A, Table IV; $\mathrm{P}<0.05$ ), suggesting that increased CXCL16 was correlated with downregulated mCD99L2 expression. In addition, in the previously established human cell lines, the expression of human CXCL16 and sCXCL16 was significantly lower in L428-CD99 ${ }^{+}$cells than in the control (Fig. 2B, Table IV; $\mathrm{P}<0.05)$, corroborating the suggestion that decreased CXCL16 is correlated with upregulated CD99 expression. 
A

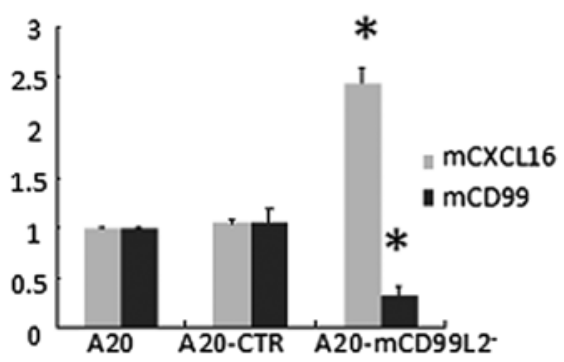

B

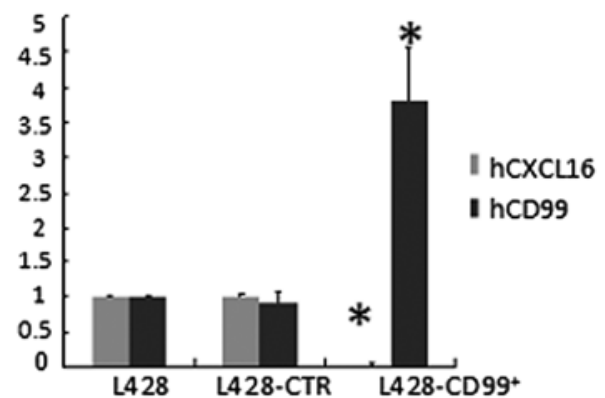

Figure 2. Relative mRNA expression of CXCL16 and CD99 by real-time RT-PCR. (A) Relative mouse CXCL16 (mCXCL16) and mouse CD99 (mCD99L2) mRNA in A20-mCD99L2- cells and control cells. (B) Relative human CXCL16 (hCXCL16) and human CD99 mRNA in L428-CD99 ${ }^{+}$cells and control cells.
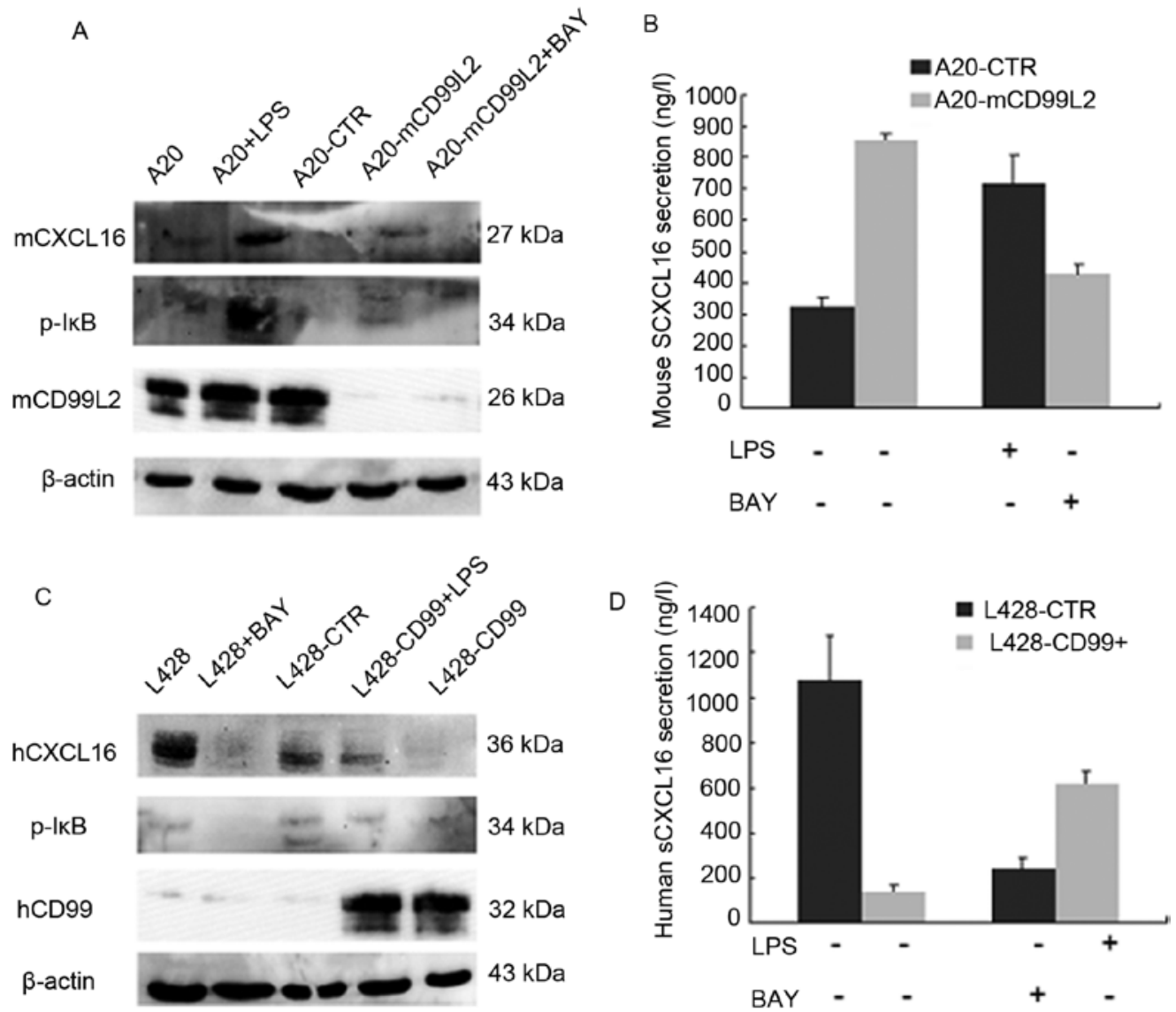

Figure 3. NF- $\mathrm{\kappa B}$ signaling is involved in the regulation of CXCL16 expression. (A) Expression of mouse CXCL16 protein (mCXCL16), phosphorylation of

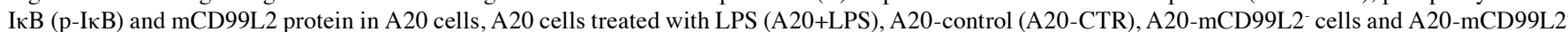
cells treated with BAY (A20-mCD99L2+BAY) were detected by western blotting, respectively. (B) ELISA results of mouse sCXCL16 secreted by A20-CTR cells, A20-mCD99L2- cell, A20-CTR cells treated with LPS and A20-mCD99L2 cells treated with BAY. (C) Western blotting results of human CXCL16 protein, phosphorylation of IкB (p-IкB) and human CD99 protein in L428 cells, L428 cells treated with BAY (L428+BAY), L428 control (L428-CTR), L428-CD99+ cell treated with LPS (L428-CD99+LPS) and L428-CD99+ cells, respectively. (D) ELISA results of human sCXCL16 secreted by L428 control cells (L428-CTR), L428-CD99+ cells, L428-CTR cells treated with BAY (L428-CTR+BAY) and L428-CD99+ cells treated with LPS (L428-CD99+ +LPS). ELISA data are presented as means \pm SD from 3 independent experiments in triplicate.

Mouse CXCL16 is affected by the NF- $\kappa B$ pathway in mouse lymphoma A20 cells as well as in the CD99 downregulated subcell type line. Subsequently, since CXCL16 is inversely correlated with human/mouse CD99 expression, the corresponding mechanism is of concern. The NF- $\mathrm{kB}$ pathway is focused on not only because of its important role in the pathology of HL, but because it can also act as a factor affecting cytokines/chemokines and $\mathrm{pI \kappa B}$, which repre- sents the activated state of the NF- $\kappa$ B pathway analyzed by western blot analysis. The inhibitor (BAY) and the activator (LPS) of the NF- $\kappa \mathrm{B}$ signaling pathway were applied and sCXCL16 was analyzed by ELISA. The results indicated that in A20-mCD99L2 cells the expression of CXCL16 protein was high and expression of $\mathrm{p}-\mathrm{I} \kappa \mathrm{B}$ protein was somewhat higher than in A20 and A20-control cells (Fig. 3A). When A20-mCD99L2 cells were treated with inhibitor BAY, 
Table IV. Secreted sCXCL16 detected by ELISA.

\begin{tabular}{lccc}
\hline Group & hCXCL16 $(\mathrm{ng} / \mathrm{l})$ & Group & mCXCL16 (ng/l) \\
\hline L428 & $1075.9 \pm 203.1$ & A20 & $323.8 \pm 28.3$ \\
L428-CTR & $1081.5 \pm 118.4$ & A20-CTR & $335.5 \pm 25.7$ \\
L428-CD99 $^{+}$ & $144.1 \pm 20.9^{\mathrm{a}}$ & A20-mCD99L2 & $854.9 \pm 23.6^{\mathrm{b}}$ \\
\hline
\end{tabular}

${ }^{\mathrm{a}} \mathrm{P}<0.05$, compared with L428 and L428-CTR cells; ${ }^{\mathrm{b}} \mathrm{P}<0.05$, compared with A20 and A20-CTR cells.

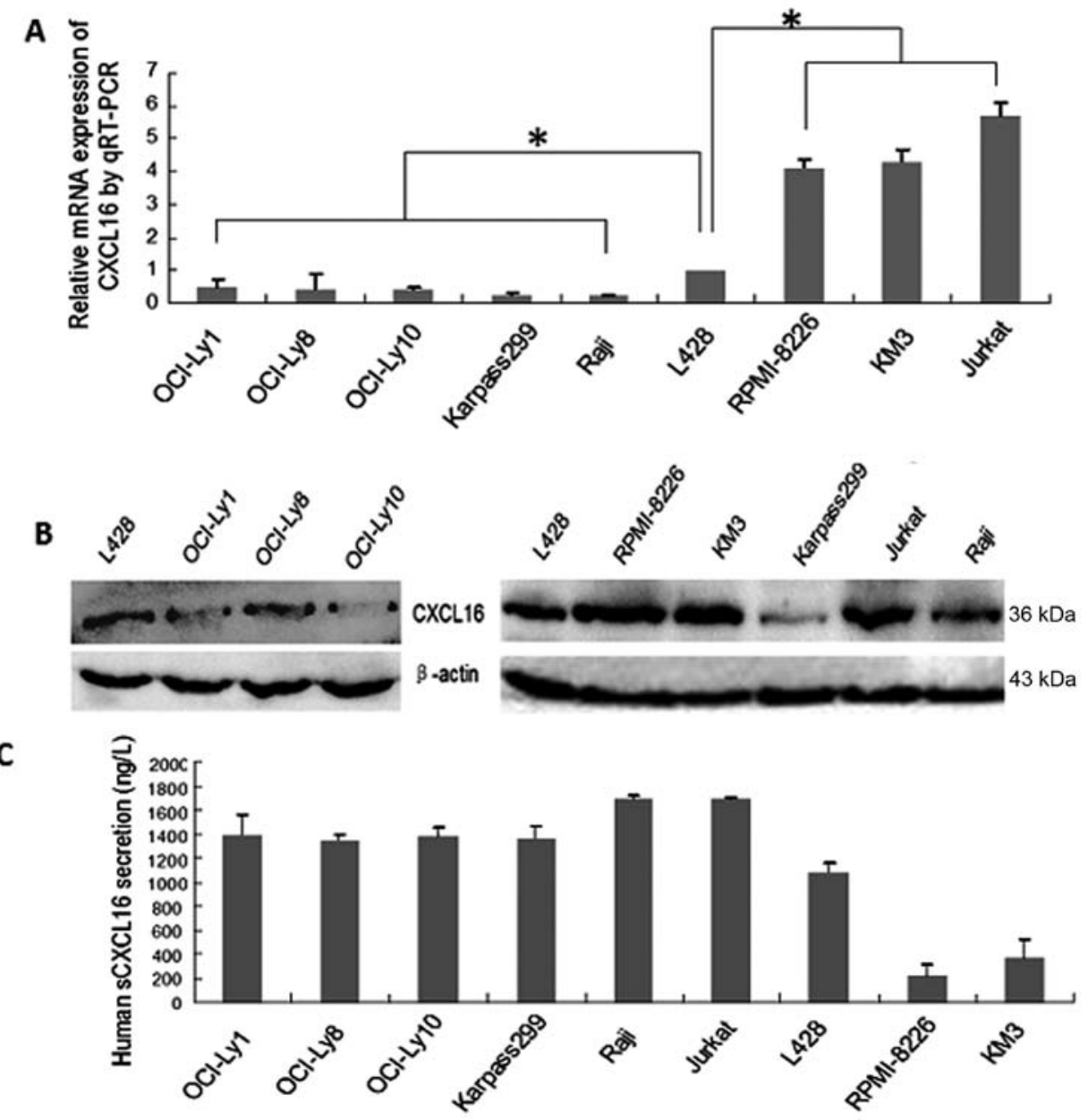

Figure 4. CXCL6 is expressed in 9 lymphoma cell lines in vitro. (A) Relative mRNA expression of CXCL6 in 9 lymphoma cell lines by quantitative RT-PCR (each group was compared with the L428 cell group which was considered as 1). (B) Expression levels of CXCL6 in 9 lymphoma cell lines by western blotting. (C) Secreted soluble CXCL16 protein in 9 lymphoma cell lines by ELISA ( $\mathrm{P}<0.05)$.

both expressions of $\mathrm{p}-\mathrm{I} \kappa \mathrm{B}$ protein and CXCL16 decreased (Fig. 3A), suggesting that inhibiting the NF- $\kappa \mathrm{B}$ pathway decreases the CXCL16 expression in the CD99 downregulated cells. By contrast, when A20 cells were treated with the activator LPS, the expression of $\mathrm{p}-\mathrm{I} \kappa \mathrm{B}$ protein and CXCL16 increased (Fig. 3A), suggesting that activating the $\mathrm{NF}-\kappa \mathrm{B}$ pathway increases the CXCL16 expression in the wild-type A20 lymphoma cells. The results from the ELISA quantification were consistent with the above data (Fig. 3B).

Human CXCL16 is affected by the NF- $\kappa B$ pathway in human L428 cells as well as in the CD99 upregulated subcell type line. In human L428-CD99+ cells, CXCL16 was lower expressed and the expression of $\mathrm{p}-\mathrm{I} \kappa \mathrm{B}$ protein was weaker than in the control (Fig. 3C).

When L428 cells were treated with the inhibitor BAY, the expression of $\mathrm{p}-\mathrm{I} \kappa \mathrm{B}$ protein and CXCL16 decreased (Fig. 3C), suggesting that inhibiting the $\mathrm{NF}-\kappa \mathrm{B}$ pathway decreases the CXCL16 expression in the CD99 upregulated cells. When L428-CD99+ cells were treated with the activator LPS, the expression of $\mathrm{p}-\mathrm{I} \kappa \mathrm{B}$ protein and CXCL16 increased (Fig. 3C), suggesting that activation of the NF- $\kappa \mathrm{B}$ pathway increases the CXCL16 expression in human L428 cells. The ELISA results were in agreement with the above findings (Fig. 3D). Taken together, our data indicated that the $\mathrm{NF}-\kappa \mathrm{B}$ signaling pathway affects the expression and secretion of CXCL16. 
A

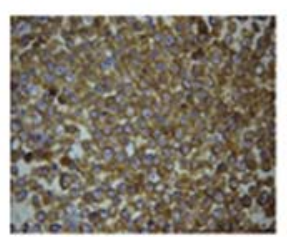

OCl-Ly10

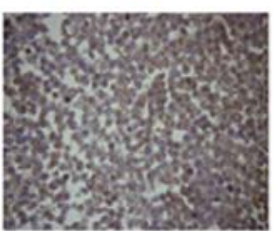

Karpass299

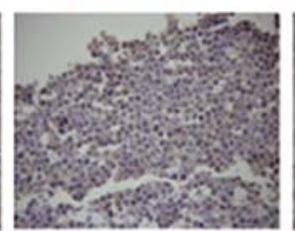

Raji
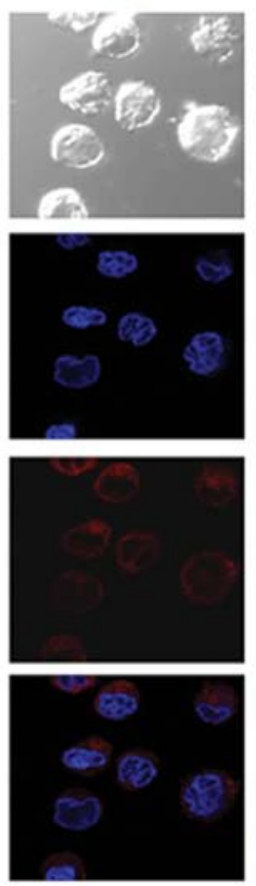

Raji

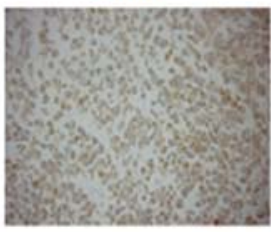

Karpass299
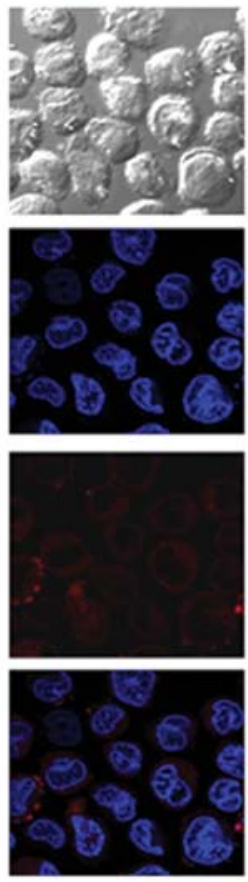

Figure 5. Morphological location of CXCL16 by ICC and confocal microscopy. (A) ICC detection of CXCL16 expression in cultured L428 cells, OCI-Ly10 cells, Karpass299 cells and Raji cells. (B) CXCL16 expression in L428 cells, OCI-Ly10 cells, Raji cells and Karpass299 cells by confocal microscopy.

CXCL16 is expressed in a series of lymphoma cell lines. Although CXCL16, including sCXCL16, were measured above and were highly expressed in human L428 as well as in mouse A20-mCD99L2- cells, CXCL16 expression in a series of lymphoma cell lines with other origins was unclear. Therefore, qRT-PCR and western blotting were used to analyze the relative expression levels of CXCL16 in 9 lymphoma cell lines. The results indicated that all 9 lymphoma cell lines expressed CXCL16 mRNA (Fig. 4A). Compared with the L428 cells, RPMI-8226, KM3 and Jurkat cells transcribed higher CXCL16 mRNA amounts, while the other 5 cell lines (OCI-Ly1, OCI-Ly8, OCI-Ly10, Karpass299 and Raji cells) transcribed relatively lower levels of CXCL16 mRNA. CXCL16 protein was expressed higher in L428, RPMI-8226, KM3 and Jurkat cells while it was expressed lower in the other 5 cell lines (Fig. 4B).

Functional sCXCL16 is secreted by different lymphoma cell lines. Since CXCL16 occurs as TM-CXCL16 and SCXCL16, the secreted sCXCL16 fractions in the 9 cell lines were analyzed by ELISA. The sCXCL16 in RPMI-8226, KM3 cell supernatants were significantly lower than in L428 cells $(\mathrm{P}<0.05)$, while Jurkat, Raji, Karpass299, OCI-Ly1, OCI-Ly8 and OCI-Ly10 cell lines secreted higher amounts of sCXCL16 than the L428 cells (Fig. 4C).
Localization of CXCL16 on the lymphoma cells. As observed in ICC experiments, specific CXCL16 protein staining was visible in the cytoplasm and membrane (TM-CXCL16) of malignant lymphoma cells (Fig. 5A). In L428 and OCI-Ly10 cells, the TM-CXCL16 was significantly predominant, while in Karpass299 and Raji cells, CXCL16 was expressed mainly in the cytoplasms. These results were also observed in the confocal microscopy analysis (Fig. 5B).

CXCL16 is expressed in different types of lymphoma samples. As the in vitro environment may be different from in vivo conditions, which involves the interaction between tumor cells and reactive immune cells, CXCL16 expressions in clinical samples of different lymphoma types were investigated. Using IHC staining, we measured the expression levels and subcellular localizations of CXCL16 protein in 80 archived paraffin-embedded lymphoma samples (Table V) as well as in 7 reactive and 7 normal lymph node sections. The results showed that the total positive rate of CXCL16 expression in the lymphomas was $68.8 \%$ (55/80); it was moderately expressed in $37.5 \%$ (30/80), strongly expressed in $31.25 \%(25 / 80)$ and negative in $31.25 \%(25 / 80)$ of the samples. In comparison, only $14.2 \%(1 / 7)$ of the 7 reactive lymph node samples and none of the normal lymph nodes expressed CXCL16 protein, which was significantly less than in the lymphoma samples $(\mathrm{P}<0.05)$. 

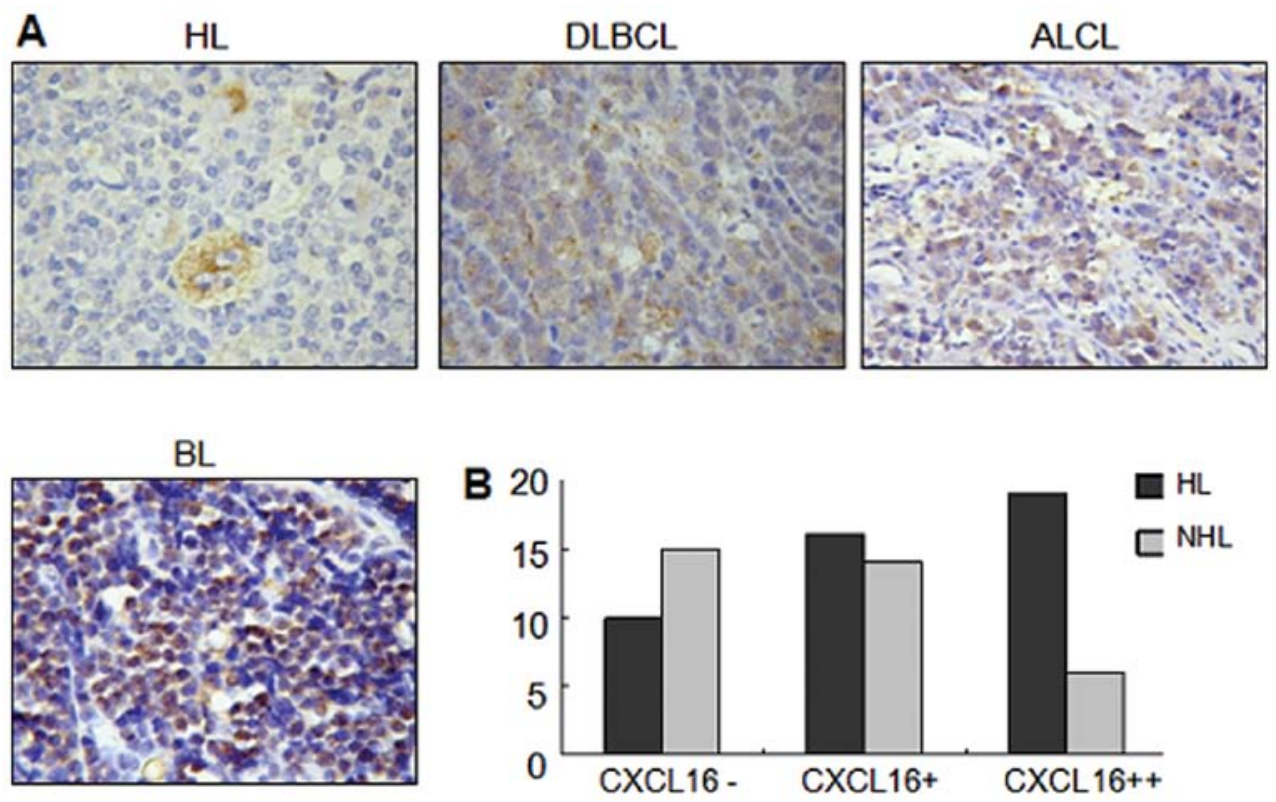

Figure 6. CXCL16 expression in clinical lymphoma samples by IHC analysis. (A) IHC analysis of CXCL16 expression in different types of lymphomas (HL, DLBCL, ALCL and BL). (B) Image of data analysis between HL and NHL samples of IHC results.

A

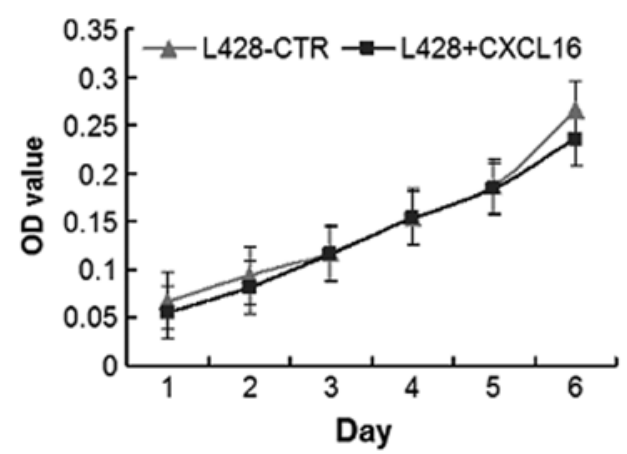

B

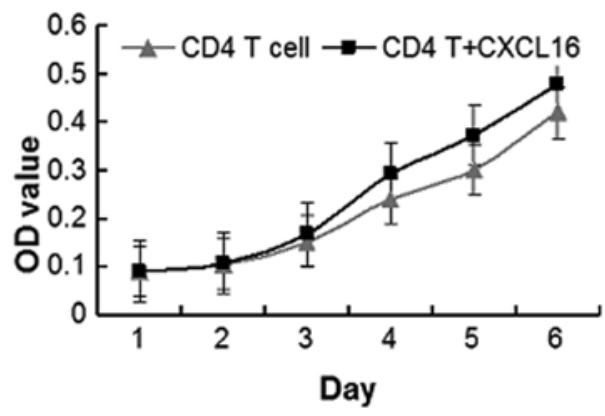

Figure 7. The proliferation of H/RS and CD4 ${ }^{+} \mathrm{T}$ cells affected by CXCL16. The proliferation ability of the human H/RS cell line (L428 cells) (A), and CD4 $\mathrm{T}$ cells from human PBMC (B) under stimulation of recombinant human CXCL16 were detected by CCK-8 proliferation assay.

In addition, the expression in HL samples was relatively higher than that in NHL (Table V, Fig. 6B; P<0.05).

Specific CXCL16 protein staining was found positive in the cytoplasm and membrane of malignant lymphoma tissues, particularly in the golgi complex where strong staining was visible (Fig. 6A). In the stromal tissues and the infiltrated inflammatory cells, CXCL16 expression was observed only in a few cells and was weak.

Proliferation of $L 428$ cells and $C D 4^{+} T$ lymphocyte under stimulation of recombinant human CXCL16. Since the expression of CXCL16 was high in HL, we hypothesized a proliferation-promoting effect of sCXCL16 in vitro, but CCK-8 proliferation assays indicated that there were no significant changes in the proliferation rates of L428 cells following incubation with recombinant human CXCL16 from day 1 to day 6 (Fig. 7A; $\mathrm{P}>0.05$ ).

Since $\mathrm{CD} 4^{+} \mathrm{T}$ cells play important roles in the composition of cHL microenvironments (24), the effect of CXCL16
Table V. Immunohistochemical analysis of CXCL16 expression in clinical lymphoma samples.

\begin{tabular}{lllll}
\hline & $\mathrm{n}$ & - & + & ++ \\
\hline $\mathrm{cHL}$ & 45 & 10 & 16 & $19^{\mathrm{b}}$ \\
$\mathrm{NHL}$ & 35 & $15^{\mathrm{a}}$ & 14 & 6 \\
\hline
\end{tabular}

${ }^{a} \mathrm{P}<0.05$, compared with $\mathrm{cHL}$; ${ }^{\mathrm{b}} \mathrm{P}<0.05$, compared with NHL. $\mathrm{cHL}$, classical Hodgkin lymphoma; NHL, non-Hodgkin lymphoma.

on the proliferation ability of $\mathrm{CD} 4^{+} \mathrm{T}$ cells in vitro was further analyzed. As a result, the proliferation rate of $\mathrm{CD} 4^{+}$ $T$ cells increased during stimulation with recombinant human CXCL16, with significant changes from day 4 to day 6 (Fig. 7B; $\mathrm{P}<0.05$ ). Our results suggested that CXCL16 might act indirectly on the H/RS cells by promoting the crosstalk between $\mathrm{CD} 4^{+} \mathrm{T}$ and $\mathrm{H} / \mathrm{RS}$ cells. 


\section{Discussion}

Several chemokines were recently reported to be expressed in tumor tissues affecting the proliferation or hemostasis of cells, particularly in inflammatory-related tumors. The role of chemokines in the biology of lymphoma has been investigated, as lymphomas are considered to be inflammatory-related tumors (25). Particularly in the cHL, the neoplastic H/RS cells construct their unique microenvironment by secreting large amounts of cytokines and chemokines $(26,27)$ and it is beneficial to elucidate the significance of the expression and pathological role of chemokines for lymphoma-related mechanisms. We focused on the CXCL16 as it was upregulated in the previously established A20-mCD99L2 cells and was extensively further upregulated in the corresponding tumor tissues in the present study, both results suggesting that there may be an association between CD99 and CXCL16. As CD99 may play a role during the differentiation of B-lymphocytes and may tend to induce the phenotypes of H/RS cells, which secrete large amounts of cytokines/chemokines to maintain their unique microenvironment, it was necessary to confirm a correlation between CXCL16 and CD99 expression. Our results indicated that the expression of CXCL16 was inversely correlated with mCD99L2 expression in murine cell lines and with CD99 in human cell lines, which provides additional insight into the role of CD99 in phenotypes of H/RS cells. The NF- $\mathrm{BB}$ pathway has been focused on due to its important role in the pathology of $\mathrm{HL}$, but also as a factor affecting cytokines/chemokines. In the present study, the effect of the NF-kB pathway on the activation of CXCL16 and CD99 revealed that NF- $\mathrm{kB}$ signaling is involved in the altered CXCL16 expression, which is inversely correlated with CD99. Since the activated NF- $\mathrm{BB}$ pathway promotes the production of several inflammatory cytokines and chemokines, CXCL16 might be a downstream target of activated NF-кB.

CXCL16 is a newly cloned chemokine which belongs to the CXC family and is expressed both as a membrane and as a transmembrane protein (TM-CXCL16) or in the supernatants in its soluble form (sCXCL16) (13). CXCL16 was found to be overexpressed in several types of cancer, such as colorectal cancer (CRC) (18), prostate cancer (19), epithelial ovarian carcinoma (20) and melanoma (21), while in pancreatic ductal adenocarcinoma patients CXCL16 is also increased in the sera (28). Preoperative high serum levels of CXCL16 were associated with metachronous liver recurrence and poor prognosis in CRC patients (30), and in prostate cancer CXCL16 production is particularly increased in aggressive cancer cells (29). Although CXCL16 has been shown to be involved in several inflammatory conditions and inflammation-related tumors, little is known about the characteristic and function of CXCL16 on lymphomas, which can be classified into several types and subtypes. The present study indicated for the first time that CXCL16 and sCXCL16 were expressed in diverse types of lymphoma cell lines. Both TM-CXC16 and sCXCL16 are highly expressed in the HL cell line (L428), which is consistent with a report of Hanamoto et al (31). sCXCL16 was highly secreted from some cell lines of $\mathrm{T}$ origin, but rather low expressed in cell lines of plasma cell origin, indicating that the secretion of CXCL16 may be different according to the origins and the differentiation states of the lymphocytes. The role of both forms during tumor progression that was only described as TM-CXCL16 seems to be a signal of good prognosis, whereas high serum sCXCL16 is a signal of poor prognosis (32). The diverse expression pattern as TM-CXCL16 or sCXCL16 in lymphomas indicated that the chemokine might play diverse roles in different types of lymphomas.

The in vitro conditions were quite different from the in vivo conditions, in which the microenvironment is affected by infiltrating lymphocytes and the tumor cells are involved in complex interactions between tumor and body immunoreactions. In the present study, we observed the expression of CXCL16 in 80 clinical lymphoma samples and found that the expression of CXCL16 was relatively higher in HL than in NHL specimens. The clinical prognosis of NHL and HL are quite different; compared with NHL, most patients with HL have a good prognosis after a therapy (33). Although there was also expression of CXCL16 in some of the NHL samples, the difference of CXCL16 expression between HL and NHL may, to some extent, reflect the different microenvironments of the two types of lymphoma. Our cell line and clinical sample research indicated a close relationship between CXCL16 expression and HL. Consistent with in vitro results in cHL ,CXCL16 was especially expressed in the neoplastic H/RS cells instead of the infiltrating lymphocytes, indicating that the tumor cells can produce and secrete the chemokine by themselves. Reports showed that CXCL16 might facilitate survival, adhesion and migration of tumor cells. For example, sCXCL16 could also promote the proliferation of adenocarcinoma (28) and may play a role in liver metastases through the induction of EMT in CRC patients (30). CXCL16 could enhance the proliferative capacity of melanoma cells by its receptor CXCR6, which was recently shown to be involved in stem cell self-renewal (21). By contrast, our results showed, that CXCL16 has no direct effect on H/RS cells, but promotes CD4 ${ }^{+}$ T-cell proliferation, which in turn might indirectly influence the survival of H/RS cells, similar to the role of radiation-induced CXCL16 in the attraction of effector T cells in breast cancer (34). Whether in HL CXCL16 may be considered a potential molecule for T-cell homing (35) requires further research of the relationship between CXCL16 cells and CD4 ${ }^{+} \mathrm{T}$ lymphocytes.

In conclusion, the present study extended our previous results by finding a correlation and signaling pathway between CD99 and CXCL16. The expressions of CXCL16 and functional sCXCL16 were investigated systematically in various cell lines and clinical samples of different origins for the first time. The diversity of CXCL16 expressions suggested a high complexity of chemokine secretions in subtypes of lymphomas. The highly expressed CXCL16 in cHL and its effect on $\mathrm{CD}^{+} \mathrm{T}$-lymphocyte proliferation provide a potential target for a biological cHL therapy.

\section{Acknowledgements}

This study was supported by a grant for the National Natural Science Foundation of China (grant nos. 81101537, 81272552 and 81071941). The authors thank Professor K.C. Chan, the Nebraska Medical Center, USA, Professor Xiaoyan Zhou, Pathology Department of Fudan University Shanghai Cancer Center, Professor Longjun Gu, Xinhua Hospital of Medicine School, Shanghai Jiao Tong University, and Department of Hematology of the Zhujiang Hospital for providing the cell lines. 


\section{References}

1. Aggarwal BB, Shishodia S, Sandur SK, et al: Inflammation and cancer: how hot is the link? Biochem Pharmacol 72: 1605-1621, 2006.

2. O'Hayre M, Salanga CL, Handel TM, et al: Chemokines and cancer: migration, intracellular signalling and intercellular communication in the microenvironment. Biochem J 409: 635-649, 2008

3. Yamashita H, Takahashi Y, Kano T, et al: Malignant lymphoma presenting as inflammation of unknown origin. Nihon Rinsho Meneki Gakkai Kaishi 35: 136-143, 2012 (In Japanese).

4. Aldinucci D, Gloghini A, Pinto A, et al: The classical Hodgkin's lymphoma microenvironment and its role in promoting tumor growth and immune escape. J Pathol 221: 248-263, 2010.

5. Küppers R: The biology of Hodgkin's lymphoma. Nat Rev Cancer 9: 15-27, 2009.

6. Kim, SH, Choi EY, Shin YK, et al: Generation of cells with Hodgkin's and Reed-Sternberg phenotype through downregulation of CD99 (Mic2). Blood 92: 4287-4295, 1998.

7. Kim SH, Shin YK, Lee IS, et al: Viral latent membrane protein1 (LMP-1)-induced CD99 down-regulation in B cells leads to the generation of cells with Hodgkin's and Reed-Sternberg phenotype. Blood 95: 294-300, 2000.

8. Huang X, Zhou X, Wang Z, et al: CD99 triggers upregulation of miR-9-modulated PRDM1/BLIMP1 in Hodgkin/Reed-Sternberg cells and induces redifferentiation. Int J Cancer 131: E382-E394, 2012.

9. Suh YH, Shin YK, Kook MC, et al: Cloning, genomic organization, alternative transcripts and expression analysis of CD99L2, a novel paralog of human CD99, and identification of evolutionary conserved motifs. Gene 307: 63-76, 2003

10. Liu F, Zhang G, Liu F, et al: Effect of shRNA targeting mouse CD99L2 gene in a murine B cell lymphoma in vitro and in vivo. Oncol Rep 29: 1405-1414, 2013.

11. Matloubian M, David A, Engel S, et al: A transmembrane CXC chemokine is a ligand for HIV-coreceptor Bonzo. Nat Immunol 1 : 298-304, 2000

12. Wilbanks A, Zondlo SC, Murphy K, et al: Expression cloning of the STRL33/BONZO/TYMSTR ligand reveals elements of CC, CXC, and CX3C chemokines. J Immunol 166: 5145-5154, 2001.

13. Hundhausen C, Schulte A, Schulz B, et al: Regulated shedding of transmembrane chemokines by the disintegrin and metalloproteinase 10 facilitates detachment of adherent leukocytes. J Immunol 178: 8064-8072, 2007.

14. Ruth JH, Haas CS, Park CC, et al: CXCL16-mediated cell recruitment to rheumatoid arthritis synovial tissue and murine lymph nodes is dependent upon the MAPK pathway. Arthritis Rheum 54: 765-778, 2006.

15. Morgan AJ, Guillen C, Symon FA, et al: Expression of CXCR6 and its ligand CXCL16 in the lung in health and disease. Clin Exp Allergy 35: 1572-1580, 2005.

16. Sheikine Y, Bang CS, Nilsson L, et al: Decreased plasma CXCL16/SR-PSOX concentration is associated with coronary artery disease. Atherosclerosis 188: 462-466, 2006.

17. Li X, Conklin L and Alex P: New serological biomarkers of inflammatory bowel disease. World J Gastroenterol 14: 5115-5124, 2008

18. Verbeke H, Struyf S, Laureys G, et al: The expression and role of CXC chemokines in colorectal cancer. Cytokine Growth Factor Rev 22: 345-358, 2011.
19. Ha HK, Lee W, Park HJ, et al: Clinical significance of CXCL16/CXCR6 expression in patients with prostate cancer. Mol Med Rep 4: 419-424, 2011.

20. Guo L, Cui ZM,Zhang J, et al: Chemokine axes CXCL12/CXCR4 and CXCL16/CXCR6 correlate with lymph node metastasis in epithelial ovarian carcinoma. Chin J Cancer 30: 336-343, 2011.

21. Taghizadeh R, Noh M, Huh YH, et al: CXCR6, a newly defined biomarker of tissue-specific stem cell asymmetric self-renewal, identifies more aggressive human melanoma cancer stem cells. PLoS One 5: e15183, 2010.

22. Darash-Yahana M, Gillespie JW,Hewitt SM, et al: The chemokine CXCL16 and its receptor, CXCR6, as markers and promoters of inflammation-associated cancers. PLoS One 4: e6695, 2009.

23. Feng MJ, Qiu C, Lai YJ, et al: Application of immunomagnetic screening strategy for separation of $\mathrm{CD} 4^{+}$and $\mathrm{CD} 8^{+} \mathrm{T}$ cell subpopulations of peripheral blood. Zhongguo Shi Yan Xue Ye Xue Za Zhi 13: 205-209, 2005 (In Chinese).

24. Zhang Y, Li XZ, Wen ZH, et al: Relationship between lymphocyte immunophenotypes and histologic subtypes of Hodgkin's lymphoma and its significance. Zhongguo Shi Yan Xue Ye Xue Za Zhi 17: 888-893, 2009 (In Chinese).

25. Bracci PM, Skibola CF, Conde L, et al: Chemokine polymorphisms and lymphoma: a pooled analysis. Leuk Lymphoma 51: 497-506, 2010.

26. Aldinucci D, Lorenzon D, Cattaruzza L, et al: Expression of CCR5 receptors on Reed- Sternberg cells and Hodgkin lymphoma cell lines: involvement of CCL5/Rantes in tumor cell growth and microenvironmental interactions. Int J Cancer 122: 769-776, 2008.

27. Hnátková M, Mociková $\mathrm{H}$, Trnený $\mathrm{M}$, et al: The biological environment of Hodgkin's lymphoma and the role of the chemokine CCL17/TARC. Prague Med Rep 110: 35-41, 2009.

28. Wente MN, Gaida MM, Mayer C, et al: Expression and potential function of the CXC chemokine CXCL16 in pancreatic ductal adenocarcinoma. Int J Oncol 33: 297-308, 2008.

29. Hu W, Zhen X, Xiong B, et al: CXCR6 is expressed in human prostate cancer in vivo and is involved in the in vitro invasion of PC3 and LNCap cells. Cancer Sci 99: 1362-1369, 2008.

30. Matsushita K, Toiyama Y, Tanaka K, et al: Soluble CXCL16 in preoperative serum is a novel prognostic marker and predicts recurrence of liver metastases in colorectal cancer patients. Ann Surg Oncol 19 (Suppl 3): S518-S527, 2012.

31. Hanamoto H, Nakayama T, Miyazato H, et al: Expression of CCL28 by Reed-Sternberg cells defines a major subtype of classical Hodgkin's disease with frequent infiltration of eosinophils and/or plasma cells. Am J Pathol 164: 997-1006, 2004.

32. Deng I, Chen N, Li Y, et al: CXCR6/CXCL16 functions as a regulator in metastasis and progression of cancer. Biochim Biophys Acta 1806: 42-49, 2010.

33. Jakovic LR, Mihaljevic BS, Perunicic Jovanovic MD, et al: The prognostic relevance of tumor associated macrophages in advanced stage classical Hodgkin lymphoma. Leuk Lymphoma 52: 1913-1919, 2011

34. Matsumura S, Wang B, Kawashima N, et al: Radiation-induced CXCL16 release by breast cancer cells attracts effector T cells. J Immunol 181: 3099-3107, 2008.

35. Machado L, Jarrett R, Morgan S, et al: Expression and function of $\mathrm{T}$ cell homing molecules in Hodgkin's lymphoma. Cancer Immunol Immunother 58: 85-94, 2009. 